\title{
ANÁLISIS DEL CRECIMIENTO HOTELERO: CASO GUARANDA PROVINCIA DE BOLÍVAR-ECUADOR
}

\author{
ANALYSIS OF HOTEL GROWTH: CASE GUARANDA PROVINCE \\ OF BOLIVAR - ECUADOR
}

\author{
María de Lourdes Larrea Paredes ${ }^{1}$ \\ llarrea@ute.edu.ec \\ Mónica Patricia Larrea Paredes ${ }^{2}$ \\ mlarrea@ecotec.edu.ec \\ Mauricio Ruiz Rojas ${ }^{1}$ \\ mauruiz1975@hotmail.com


Para citar este artículo:

Larrea P. María, Larrea M., Ruiz M. (2020). Análisis del crecimiento hotelero: caso guaranda

provincia de Bolívar-Ecuador. Espacio I+D, Innovación más Desarrollo. IX(23), 8-19. doi: http://dx.doi.org/10.31644/IMASD.23.2020.a01

\section{RESUMEN}

En este documento se realiza un análisis comparativo del crecimiento de la planta hotelera en la ciudad de Guaranda, Provincia Bolívar - Ecuador en el periodo 2009-2017. El objetivo es determinar el crecimiento hotelero y el impacto que ha generado en el porcentaje de ocupación de las empresas de alojamiento. La metodología utilizada fue de tipo cualitativo - cuantitativo, se realizó investigación de campo para la obtención de información utilizando encuestas en los establecimientos hoteleros existentes en la ciudad de Guaranda, se elaboraron entrevistas que fueron aplicadas a los propietarios de estos. Se determinó que la ciudad de Guaranda ha tenido un crecimiento en número de establecimientos y en plazas, lo cual ha generado que los porcentajes de ocupación de los hoteles estén en un nivel bajo comparada con la capacidad instalada.

\section{Palabras clave}

Guaranda, hotelería, planta hotelera, porcentaje de ocupación. 
This research presents a comparative analysis of the growth of the hotel plant in the city of Guaranda, Bolivar Province - Ecuador in the period 2009-2017. The objective is to determine hotel growth and the impact it has generated on the percentage of occupancy of accommodation companies. The methodology used was qualitative - quantitative, field research was conducted to obtain information using surveys in existing hotel establishments in the city of Guaranda, and interviews were developed that were applied to their owners. It was determined that the city of Guaranda has had a growth in the number of establishments and places, which has resulted in the hotel occupancy rates being low compared to the installed capacity.

\section{Keywords}

Guaranda, hotel, hotel plant, occupancy percentage. 
L

a ciudad de Guaranda se encuentra ubicada en la Provincia Bolívar, Ecuador, en el centro-sur de la región interandina, fundada por los españoles en 1571, elevada a Villa Mayor el 11 de noviembre de 1811, el 9 de noviembre de 1820 sufrió la batalla de Camino Real, donde las tropas de la corona fueron derrotadas y Guaranda proclamó su Independencia del dominio español. Fue declarada Patrimonio Cultural del Ecuador el 23 de octubre del 1997 (Gobierno Autónomo Descentralizado Guaranda, 2018).

\section{HISTORIA DE LA HOTELERÍA EN LA CIUDAD DE GUARANDA}

Guaranda era el paso entre la costa y la sierra por la antigua Vía Flores, y era una próspera población. Existían familias que alojaban a los viajeros y comerciantes que iban a Bodegas de Babahoyo, hoy ciudad, y otras poblaciones del litoral. Transitaban con recuas de mulares que traían hielo del nevado Chimborazo y productos de la sierra; estos pernoctaban en lugares conocidos como Tambos.

Se conoce por referencias de pobladores de la zona que los albergues existentes se ubicaron en sitios estratégicos como Las Herrerías, Vinchoa, San Lorenzo, Santiago, Bilován lugar donde se ubicaba el Tambo de Angas. La importancia de este Tambo la confirmó Don Pedro Vicente Maldonado en su carta (mapa) de la provincia de Quito, sacada a la luz por orden y a expensas de su Majestad Luis XV, en 1750.

El escritor ambateño Luis A. Martínez en su obra "A la Costa", describe con lujo de detalles el paso por los parajes bolivarenses, así menciona: "Ya muy entrada la tarde llegó el viajero a Balzapamba, el primer pueblo, o más bien caserío de tierra caliente, en el camino que va de Guaranda a Babahoyo", narra también el lugar donde pernoctaría para tomar el descanso y alimentación, "desmóntese el viajero delante de una casita mal llamada hotel. El arriero que llevaba equipaje en un caballito flaco y lanudo, descargole, y subiendo empinada escalera, pidió para el patrón cuarto y comida. Un hombre pálido, mal encarado, condujo al huésped a un zaquizamí para que guardara el pobre equipaje" (Martinez, 1969, págs. 146-147)

Se tiene referencia, por personas de la ciudad de Guaranda, que existieron en la capital de la provincia algunas casas que brindaban hospedaje a los viajeros, se mencionan las siguientes: casa posada del señor Gregorio Coloma, cuya existencia data del siglo XIX, se manifiesta que aquí se hospedó el Dr. Gabriel García Moreno ex presidente del Ecuador, se desconoce el año que dejó de ofrecer servicio de alojamiento.

Casa Posada del señor Benjamín Lombeida, se conocía con el nombre de Tambo, ofrecía hospedaje y servía para intercambiar productos de costa y sierra. Aproximadamente en el año 1918 entra en funcionamiento la casa posada de la señora Joaquina Dávila ubicada en las calles García Moreno 
y Convención de 1884. Alrededor de 1938 comienza a prestar servicio de alojamiento la casa posada Central de la Sra. Mercedes de del Salto localizada en la calle Convención de 1884. Posteriormente en el año 1938 se instala en Guaranda la primera pensión con el nombre "Ecuador" de propiedad de la señora Rosa Espinoza de Vásconez, que brindaba servicio de alojamiento en habitaciones simples, dobles y triples. En el año 1940 abre sus puertas el hotel Ramírez localizado en las calles Azuay y Sucre, dejó de funcionar en el año 1960.

Las casas posadas fueron desapareciendo debido a la construcción de la vía del ferrocarril, que unió la sierra con la costa y que por conveniencia política y económica fue trazada por zonas como Alausí, Sibambe y la Nariz del Diablo, y no por la vía Kelly en la Provincia de Bolívar. (Larrea, 1998)

En el año 1968, aproximadamente, entra en funcionamiento la pensión Tequendama, luego se crearon otros alojamientos como el Hotel Cochabamba, operando a partir del año 1970, en el año 1986 cambia de administración, contando desde su inicio con servicios de hospedaje y alimentación.

En el año 1972 el Hotel Bolívar brinda a sus clientes habitaciones con baño privado y alimentación. En 1978 comienza la construcción el Hotel la Colina, que entra en funcionamiento en 1979, ofreciendo servicios de alojamiento, alimentación, piscina, sauna, canchas de tenis, vóley, bar, salón de eventos y otros productos complementarios.

Un grupo de entusiastas personajes bolivarenses, conjuntamente con el Consejo Provincial y el Municipio de Guaranda, observó que la provincia y la ciudad contaban con un solo hostal de primera categoría para hospedar a turistas nacionales y extranjeros, emprendieron la construcción del Hotel Tambo el Libertador, mismo que por falta de apoyo económico al área turística no se llegó a culminar y quedó abandonada por varios años, hasta que en el año 2002 se completó su edificación y equipamiento abriendo sus puertas al público.

"Guaranda posee una superficie de 1897,8 Km2 está una altura de 2668 msnm y un clima templado el cual varía desde los fríos de páramo debido a su cercanía con el nevado Chimborazo, hasta un temperado subtropical del valle”. (Guerrón \& Terán , 2014), posee diferentes atractivos turísticos naturales y culturales como se muestran en la Tabla 1. 


\section{Tabla 1}

Atractivos del Cantón Guaranda

\begin{tabular}{|c|c|}
\hline \\
\hline ATRACTIVOS NATURALES & ATRACTIVOS CULTURALES \\
\hline El Peñón y Complejo Camilo Montenegro & Carnaval de Guaranda \\
\hline Mirador Natural Colina San Bartolo & Centro Histórico de la ciudad de Guaranda \\
\hline Mirador Natural Colina Cruz Loma & Mirador y Centro Cultural Indio Guaranga \\
\hline Mirador Natural Colina San Jacinto & Pase del Niño en la ciudad de Guaranda \\
\hline Mirador Natural Colina Talalag & La Feria y Mercados de ciudad de Guaranda \\
\hline Mirador Natural Colina Tililag & Museo del Carnaval \\
\hline Mirador Loma de Guaranda & El Día de los Difuntos \\
\hline Mirador Natural Colina El Calvario & $\begin{array}{l}\text { Fiestas por San Pedro y San Pablo en la parroquia de } \\
\text { Guanujo }\end{array}$ \\
\hline Bosque de Polylepís & $\begin{array}{l}\text { Artesanía, Elaboración de Tejidos de Lana, Quesos y } \\
\text { otros productos en la parroquia de Salinas }\end{array}$ \\
\hline El Troje, Orillas y Cascada del Río Salinas & Fiesta de los Santos Reyes en la parroquia de Salinas \\
\hline Cascada de Ishpingo & Ruinas Arqueológicas de Guanguliquín \\
\hline Complejo Ecológico Los Caseiches & La Gruta del Padre Santo \\
\hline El Arenal y Paisaje Natural & Andinismo al Chimborazo \\
\hline Laguna Las Cochas, (Puricocha y Patococha) & Piedras Arqueológicas y Cuevas \\
\hline Bosque de Peña Blanca & Artesanía, Elaboración de varios productos \\
\hline Reserva Faunística Chimborazo & Bandas de Juegos, Música, Juegos Populares \\
\hline \multicolumn{2}{|l|}{ Minas de Sal } \\
\hline \multicolumn{2}{|l|}{ Laguna Cocha Colorada } \\
\hline \multicolumn{2}{|l|}{ Rápidos los Caseiches } \\
\hline \multicolumn{2}{|l|}{ Cuevas De Tiagua } \\
\hline $\begin{array}{l}\text { Yacimientos Mineros en las parroquias de } \\
\text { Salinas y Simiatug }\end{array}$ & \\
\hline
\end{tabular}

Fuente: Gobierno Autónomo Descentralizado Guaranda. Elaborado por: Autores

El Carnaval de Guaranda representa la mayor fiesta de la ciudad y con Acuerdo Ministerial No. 4291 del Ministro de Educación y Cultura Deportes y Recreación del 31 de octubre del 2002, fue declarado Patrimonio Cultural Inmaterial del Estado Ecuatoriano. Se realiza entre febrero o marzo de cada año, esta festividad convierte a Guaranda en una de las ciudades más visitadas tanto por turistas nacionales y extranjeros.

Durante mayo, la ciudad es visitada durante la realización de las fiestas de fundación de la provincia, caracterizada por la realización de ferias y exposiciones que motivan a turistas y comerciantes a visitar la ciudad. 


\section{DESARROLLO}

\section{Reglamentación hotelera del Ecuador}

El sector turístico y hotelero en el Ecuador ha sido regulado por instituciones de gobierno como la Corporación Ecuatoriana de Turismo y Ministerio de Turismo, estas entidades han generado normativa que ha permito clasificar a los establecimientos de acuerdo a su infraestructura, personal, servicio que ofertan entre otras, estos reglamentos fueron los siguientes:

- Reglamento Hotelero del Ecuador, publicado en el Registro Oficial $\mathrm{N}^{\circ} .699$ del 26 de octubre de 1978

- Reglamento General de Actividades Turísticas, publicado en el Registro Oficial No. 726 de 17 de diciembre del 2002.

- $\quad$ Reglamento de Actividades Turísticas Registro Oficial No 465 del 24 de marzo del 2015.

La tabla 2 contiene la información relativa a la clasificación de los establecimientos hoteleros, estas categorizaciones permiten identificar las diferencias existentes en la denominación de los establecimientos de alojamiento, mismas que disponen de categorías de acuerdo a la infraestructura, personal y otros elementos que poseen los mismos. 
Tabla 2

Clasificación los establecimientos de alojamiento

\begin{tabular}{|c|c|c|}
\hline Reglamento Hotelero 1978 & Reglamento hotelero 2002 & $\begin{array}{c}\text { Reglamento de actividades } \\
\text { turísticas } 2015\end{array}$ \\
\hline Alojamientos hoteleros & Alojamientos hoteleros & Hotel \\
\hline \multirow[t]{2}{*}{ Hoteles } & Hoteles & Hostal \\
\hline & Hotel & \\
\hline Hotel Residencia & Hotel Residencia & Hostería \\
\hline Hotel Apartamento & Hotel Apartamento & Hacienda turística \\
\hline Hostales y pensiones & Hostales y pensiones & Lodge \\
\hline \multirow[t]{2}{*}{ Hostales } & Hostales & Resort \\
\hline & Hostales residencias & \\
\hline Pensiones & Pensiones & Refugio \\
\hline $\begin{array}{l}\text { Hosterías, moteles, refugios y } \\
\text { cabañas }\end{array}$ & $\begin{array}{l}\text { Hosterías, moteles, refugios y } \\
\text { cabañas }\end{array}$ & Campamento Turístico \\
\hline Hosterías & Hosterías & Casa de huéspedes \\
\hline Moteles & Moteles & \\
\hline Refugios & Refugios & \\
\hline Cabañas & Cabañas & \\
\hline Alojamientos no Hoteleros & Alojamientos no Hoteleros & \\
\hline Ciudades vacacionales & Complejos vacacionales & \\
\hline Campamentos & Campamentos & \\
\hline Apartamentos & Apartamentos & \\
\hline
\end{tabular}

Fuente: Elaboración propia

En este análisis se observa el cambio de clasificación de los establecimientos de alojamiento de acuerdo a los reglamentos del sector Hotelero de 1978 y 2002, en los cuales se establece la misma clasificación, en el reglamento del 2015 se evidencian modificaciones importantes, en donde se suspenden las clasificaciones de establecimientos hoteleros y no hoteleros y se nombran a ciertos establecimientos como lodge, resort, haciendas turísticas y casas de huéspedes, desapareciendo los moteles, que actualmente son regulados por las Intendencias de Policía.

Esta investigación se realizó a partir de fuentes primarias y secundarias que permitieron la revisión y análisis, es de tipo cuantitativa-cualitativa que permitió la recopilación de datos, análisis y organización, tiene un alcance descriptivo, ya que se recolectó la información en relación a los establecimientos de alojamiento de la ciudad de Guaranda con entrevistas realizadas a pobladores de la ciudad que conocen la evolución del hospedaje en la ciudad. 


\section{RESULTADOS Y DISCUSIÓN}

En la Figura 1. Se aprecia que el principal motivo de viaje de las personas que visitan Guaranda es por negocios, seguidos por el turismo y descanso. Según información proporcionada por la Dirección de Turismo del Gobierno Descentralizado del cantón Guaranda, los meses de febrero, marzo y abril son los meses de mayor visita de turistas a la ciudad.

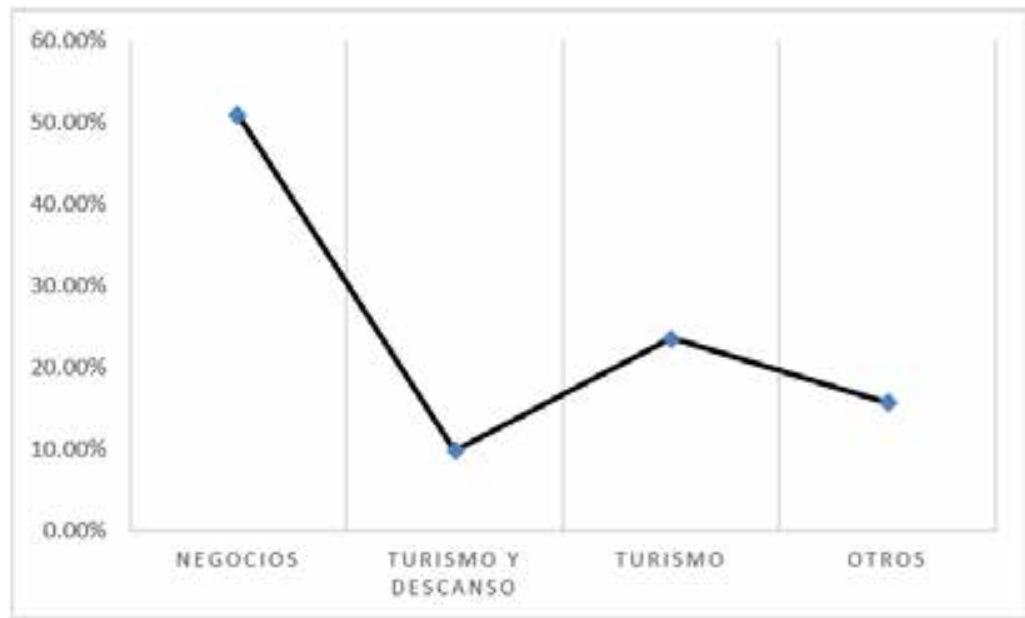

Figura 1. Motivo de vista a la Ciudad de Guaranda. Elaborado por los autores

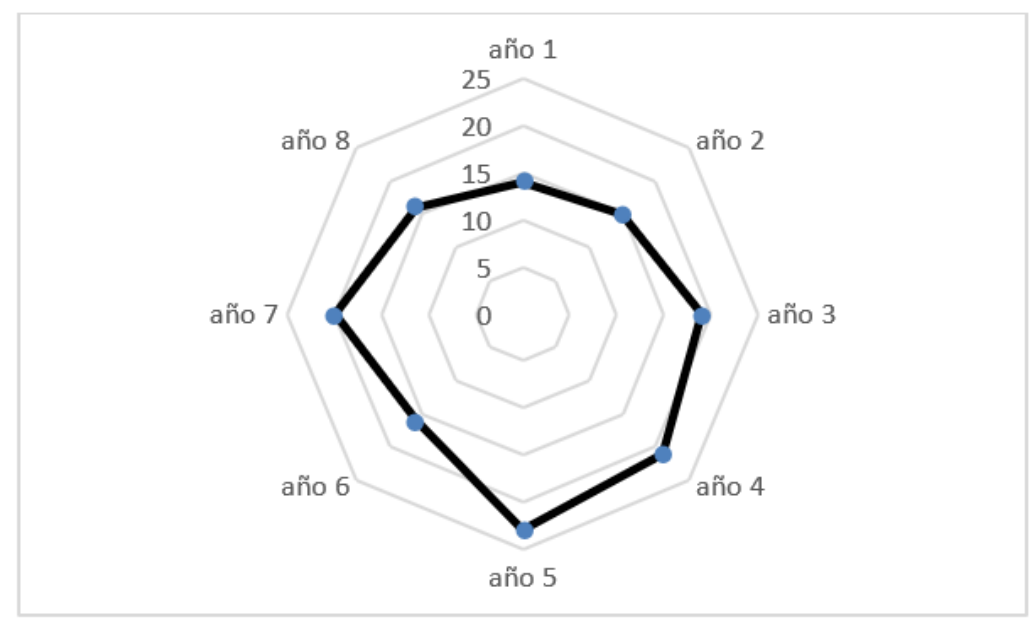

Figura 2. Establecimientos existentes en la ciudad de Guaranda. Elaborado por los autores

En la Figura 2, el quinto y séptimo año se puede apreciar que presentan un mayor crecimiento de establecimientos. En la tabla 3, se puede observar que en la ciudad de Guaranda los establecimientos de alojamiento existentes entre los años 5 y 6 presentan un decrecimiento del $-43.75 \%$ esto debido a 
que en el Reglamento de Actividades Turísticas que entra en vigencia en el 2015 no considera a los moteles como parte de la clasificación.

\section{Tabla 3}

Establecimientos existentes en la ciudad de Guaranda

\begin{tabular}{ccc}
\hline Años & $\begin{array}{c}\text { Número de establecimientos } \\
\text { registrados MINTUR }\end{array}$ & Porcentaje de variación \\
año 1 & 14 & \\
año 2 & 15 & 6.67 \\
año 3 & 19 & 21.05 \\
año 4 & 21 & 9.52 \\
año 5 & 23 & 8.70 \\
año 6 & 16 & -43.75 \\
año 7 & 20 & 20.00 \\
año 8 & 16 & -25.00 \\
\hline
\end{tabular}

Fuente: Catastros MINTUR 2012, 2013, 2014, 2015, 2016, 2017. Elaboración propia

Se presenta, en la Tabla 4, la capacidad instalada en relación al número de plazas de los establecimientos de alojamiento de la ciudad de Guaranda.

\section{Tabla 4}

Habitaciones y plazas existentes en la ciudad de Guaranda

\begin{tabular}{cccc}
\hline Año & Habitaciones (diario) & $\begin{array}{c}\text { Plazas/ } \\
\text { Habitaciones (diario) }\end{array}$ & $\begin{array}{c}\text { Capacidad máxima } \\
\text { instalada (pax anual) }\end{array}$ \\
año 1 & 172 & 319 & 116435 \\
año 2 & 153 & 183 & 66795 \\
año 3 & 342 & 754 & 275210 \\
año 4 & 370 & 834 & 304410 \\
año 5 & 330 & 718 & 262070 \\
año 6 & 281 & 662 & 241630 \\
año 7 & 329 & 732 & 267180 \\
año 8 & 274 & 608 & 221920 \\
\hline
\end{tabular}

Fuente: Elaboración propia a partir de catastros de alojamientos turísticos del Ministerio de Turismo

Aplicando la fórmula de la Figura 3. se pudo determinar que el crecimiento anual de los establecimientos hoteleros fue del 9.65\%.

$$
T C A=\left(\sqrt[N-1]{\frac{V f}{V i}}-1\right) \cdot 100
$$

Figura 3. Tasa de crecimiento acumulada 
Según estadísticas proporcionadas por el Gobierno Descentralizado del Cantón Guaranda, se tuvo un ingreso de turistas en el año 7 de 14738 turistas (que se hospedaron en establecimientos de alojamiento). Si se realiza un comparativo de la capacidad instalada en el mismo año, se pudo determinar que el porcentaje de ocupación es de $5.51 \%$. Para obtener este dato se aplicó la fórmula de la Figura 4.

$$
P O=\frac{N^{\circ} \text { de plazas } * 100}{\text { Capacidad Máxima }}
$$

Figura 4. Porcentaje de ocupación

\section{CONCLUSIONES}

La ciudad de Guaranda, a pesar de contar con atractivos naturales, tiene como actividad secundaria el turismo. La planta hotelera existente brinda la oportunidad de ofertar servicios de alojamiento, sin embargo es importante generar una promoción turística fundada en datos objetivos, que fomente la visita a esta ciudad.

En la ciudad de Guaranda no se cuenta con datos estadísticos que puedan contribuir a generar planes de desarrollo turístico, que permitan determinar la procedencia de los turistas, para establecer planes que fomenten la visita tanto de turistas nacionales como internacionales a esta ciudad.

En la ciudad existen establecimientos de alojamiento que no se encuentran registrados en los organismos encargados de regular el turismo, por lo cual la capacidad, en cuanto al número de habitaciones y plazas, que pueden recibir a los visitantes, es superior a la estimada, lo que genera una sobre oferta, comparada con el número de turistas y comerciantes que visitan la ciudad de Guaranda, por lo que se hace necesario que instituciones como el Ministerio de Turismo, el Gobierno Descentralizado del Cantón Guaranda y la Cámara provincial de Turismo de Bolívar, regulen la creación y operación de dichos establecimientos. 


\section{REFERENCIAS}

Gobierno Autónomo Descentralizado Guaranda. (2018). Gobierno Autónomo Descentralizado Cantón Guaranda. Obtenido de Gobierno Autonomo Descentralizado Cantón Guaranda: www.guaranda.gob.ec/ newsitecмт/historia/

Guerrón, A., \& Terán, S. (2014). Guranda lugares y memorias. Quito: Graficas Ayerve.

Larrea, M. L. (1998). Evolución y Perspectiva del desarrollo hotelero en la provincia Bolívar con el fin de dar una adecuada promoción y publicidad (Tesis de Pregrado). Quito: Universidad Tecnológica Equinoccial.

Martinez, L. (1969). A la Costa. Quito: Casa de la Cultura Ecuatoriana. 